\title{
Optimal Dynamic Proportional and Excess of Loss Reinsurance under Dependent Risks
}

\author{
Cristina Gosio, Ester C. Lari, Marina Ravera \\ Department of Economics and Business Studies, University of Genoa, Genoa, Italy \\ Email: gosio@economia.unige.it, lari@economia.unige.it, ravera@economia.unige.it
}

Received 11 May 2016; accepted 12 June 2016; published 15 June 2016

Copyright (C) 2016 by authors and Scientific Research Publishing Inc.

This work is licensed under the Creative Commons Attribution International License (CC BY). http://creativecommons.org/licenses/by/4.0/

(c) () Open Access

\begin{abstract}
In this paper, we study an optimal reinsurance strategy combining a proportional and an excess of loss reinsurance. We refer to a collective risk theory model with two classes of dependent risks; particularly, the claim number of the two classes of insurance business has a bivariate Poisson distribution. In this contest, our aim is to maximize the expected utility of the terminal wealth. Using the control technique, we write the Hamilton-Jacobi-Bellman equation and, in the special case of the only excess of loss reinsurance, we obtain the optimal strategy in a closed form, and the corresponding value function.
\end{abstract}

\section{Keywords}

Reinsurance, Proportional Reinsurance, Excess of Loss Reinsurance, Hamilton-Jacobi-Bellman Equation

\section{Introduction}

In the last two decades the optimal reinsurance problem has had an important impact in the actuarial literature. Several authors have studied this problem with different purposes and referring to different surplus processes. Starting from the classical model where the process of the total claim amount has a Poisson compound distribution or follows a diffusion process, the adjustment coefficient, or the expected utility of the terminal wealth are been optimized (see, for example, [1] and [2]).

With similar optimization aims, a more realistic model has been often considered, with two or more dependent classes of insurance business. Similar approaches are, for example: in [3] where the excess of loss insurance is considered and the adjustment coefficient or the expected utility of the terminal wealth are maximized, in [4] and in [5] where the expected utility of the terminal weal this maximized, in [6] where the adjustment coefficient is maximized. This paper considers two classes of insurance business, dependent through the number of 
claims, and considers the proportional and the excess of loss reinsurances. The paper is organized as follows: in Section 2 the assumptions and the model are explained, in Sections 3 and 4, the problem is presented; subsequently the Hamilton-Jacobi-Bellman (HJB) equation is given and discussed in some particular cases. In Section 5 , the problem with the only excess of loss reinsurance is solved; the optimal strategy and the corresponding value function are obtained.

\section{The Model}

We consider the finite time horizon $[0, T], 0<T<\infty$ and a model in which two dependent risks are involved. In particular, we assume two classes of insurance business, being the claim number processes correlated. The arrival claim processes are $\left\{N_{i}(t), i=1,2 ; t \in[0, T]\right\}$ we assume that these processes are Poisson processes defined as follows:

$$
N_{i}(t)=Q_{i}(t)+Q_{12}(t), i=1,2, t \in[0, T],
$$

where $Q_{1}, Q_{2}$ and $Q_{12}$ are Poisson random variables with positive parameters $\theta_{1}, \theta_{2}$ and $\theta_{12}$ respectively.

We furthermore assume that $X_{i j}, j=1,2, \cdots$, are the random variables claim size of the risks $i, i=1,2$, where we assume that $X_{1 j}$ and $X_{2 j}$ have the same distribution functions $F_{1}$ and $F_{2}$ with $F_{i}(x)=0$, for $x \leq 0$, and expected value $E\left[X_{i j}\right]=\mu_{i}<+\infty, i=1,2$. We also assume that the moment generating functions:

$$
M_{X i j}(r), i=1,2, j=1,2, \cdots,
$$

exist. As usually stated, the random variables $\left\{X_{i j}, i=1,2, j=1,2, \cdots\right\}$, are mutually independent, and independent of $\left\{N_{i}(t), i=1,2 ; t \in[0, T]\right\}$.

Let $S_{i}(t), t \in[0, T], i=1,2$, the aggregate claims amounts for the two classes of insurance risk. Because of the made assumptions, the process $\left\{\left(N_{1}(t), N_{2}(t)\right) ; t \in[0, T]\right\}$, has a bivariate Poisson distribution and $S_{1}(t)$ and $S_{2}(t)$ are correlated by $\theta_{12}$ resulting:

$$
S_{i}(t)=\sum_{j=1}^{N_{i}(t)} X_{i j}, i=1,2
$$

We consider the random variables $X_{i}, i=1,2$, identically distributed to $X_{i j}, j=1,2, \cdots$, respectively. We assume that the random variables $X_{i}$ are upper limited or that $\lim _{s \rightarrow+\infty}\left[1-F_{i}(s)\right] s=0$.

We denote by $c_{i}, i=1,2$, the premium rate, calculated by the expected value principle, including a safety loading coefficient $\eta_{i}$ :

$$
c_{i}=\left(1+\eta_{i}\right)\left(\theta_{i}+\theta_{12}\right) \mu_{i}, \eta_{i}>0, i=1,2
$$

We assume that the principal insurer can implement both a proportional and an excess of loss reinsurance referred to both classes of insurance risks, with the respective retention levels $a_{i}(t) \in(0,1), i=1,2$ for the proportional reinsurance, and retention limits $b_{i}(t) \in(0,+\infty), i=1,2$, for the excess of loss reinsurance.

We therefore denote by $\left\{\left(a_{1}(t), a_{2}(t), b_{1}(t), b_{2}(t)\right), t \geq 0\right\}$ any admissible control strategy that, for simplicity, we denote by $(a, b)$.

The reinsurer, because of the proportional reinsurance, would pay $\left(1-a_{i}\right) X_{i}$ for each claim of $i$-type; however, because of the further excess of loss reinsurance, he pays:

$$
\begin{cases}\left(1-a_{i}\right) X_{i} & \text { if } a_{i} X_{i} \leq b_{i} \\ \left(1-a_{i}\right) X_{i}+a_{i} X_{i}-b_{i}=X_{i}-b & \text { if } a_{i} X_{i} \geq b_{i}, i=1,2\end{cases}
$$

that is:

$$
Z_{i}=\left\{\begin{array}{cc}
\left(1-a_{i}\right) X_{i} & \text { if } X_{i} \leq \frac{b_{i}}{a_{i}} \\
X_{i}-b_{i} & \text { if } X_{i} \geq \frac{b_{i}}{a_{i}}
\end{array}\right.
$$

We assume that all the premiums are paid using the expected value principle. Therefore, the reinsurance premium rate at time $t$ is, for each class of risk: 


$$
P_{i}\left(a_{i}, b_{i}\right)=\left(1+\gamma_{i}\right)\left(\theta_{i}+\theta_{12}\right) \mathrm{E}\left[Z_{i}\right], \gamma_{i}>\eta_{i}, i=1,2,
$$

where we have assumed the safety loading coefficients $\gamma_{i}>\eta_{i}$, that is:

$$
P_{i}\left(a_{i}, b_{i}\right)=\left(1+\gamma_{i}\right)\left(\theta_{i}+\theta_{12}\right)\left\{\mu_{i}-a_{i} \int_{0}^{\frac{b_{i}}{a_{i}}}\left(1-F_{i}(s)\right) \mathrm{d} s\right\} .
$$

Therefore, after the reinsurances, the premium rate for the insurer is:

$$
P:=\sum_{i=1}^{2}\left(c_{i}-P_{i}\left(a_{i}, b_{i}\right)\right)=\sum_{i=1}^{2}\left[\left(\theta_{i}+\theta_{12}\right) \mu_{i}\left(\eta_{i}-\gamma_{i}\right)+\left(1+\gamma_{i}\right)\left(\theta_{i}+\theta_{12}\right) a_{i} \int_{0}^{\frac{b_{i}}{a_{i}}}\left(1-F_{i}(s)\right) \mathrm{d} s\right] \text {. }
$$

\section{The Problem}

We assume that the insurer can choose, for every time $t, t \in[0, T]$, the $a_{i}(t)$ and $b_{i}(t), i=1,2$, according to the observable information about the insurance risk processes up to time $t$. This means that $a_{i}(t)$ and $b_{i}(t), i=1,2$, are the control parameters that allow us to consider the following set of admissible strategies:

$$
A=\left\{\left(a_{1}(t), a_{2}(t), b_{1}(t), b_{2}(t)\right)=(a, b) ; a_{i}(t) \in(0,1), b_{i}(t) \in[0,+\infty), t \in[0, T]\right\}
$$

The main goal for the insurer is to choose an optimal reinsurance strategy that maximize the expected exponential utility of terminal wealth. To solve this problem, we will use a dynamic programming approach.

After the reinsurance, remembering (4), referring to the $j$-th claim of type $i$, the insurer pays $\bar{X}_{i j}$

$$
\bar{X}_{i j}= \begin{cases}a_{i} X_{i} & \text { if } X_{i} \leq \frac{b_{i}}{a_{i}} \\ b_{i} & \text { if } X_{i} \geq \frac{b_{i}}{a_{i}} .\end{cases}
$$

Hence, the total claim amount charged to the insurer at time $t$, referred to the $i$-type claim is:

$$
\bar{S}_{i}(t)=\sum_{j=1}^{N_{i}(t)} \bar{X}_{i j}, i=1,2
$$

It follows that the surplus process $R(t)$ controlled by the reinsurance strategies, evolves over the time as follows:

$$
\begin{aligned}
\mathrm{d} R(t)= & \sum_{i=1}^{2}\left[\left(\theta_{i}+\theta_{12}\right) \mu_{i}\left(\eta_{i}-\gamma_{i}\right)+\left(1+\gamma_{i}\right)\left(\theta_{i}+\theta_{12}\right) a_{i} \int_{0}^{\frac{b_{i}}{a_{i}}}\left(1-F_{i}(s)\right) \mathrm{d} s\right] \mathrm{d} t \\
& -\mathrm{d}\left[\bar{S}_{1}(t)+\bar{S}_{2}(t)\right]=P \mathrm{~d} t-\mathrm{d}\left[\bar{S}_{1}(t)+\bar{S}_{2}(t)\right] .
\end{aligned}
$$

We recall that the process $\left\{\left(N_{i}(t), i=1,2 ; t \in[0, T]\right\}\right.$ has a bivariate Poisson distribution with stationary increments; therefore, using results in [3] and [5], it results that:

$$
\begin{aligned}
& {\left[\bar{S}_{1}(t+\mathrm{d} t)+\bar{S}_{2}(t+\mathrm{d} t)\right]-\left[\bar{S}_{1}(t)+\bar{S}_{2}(t)\right]} \\
& = \begin{cases}0 & \text { with probability } 1-\left(\theta_{1}+\theta_{2}+\theta_{12}\right) \mathrm{d} t \\
\min \left(a_{1} X_{1}, b_{1}\right):=Y_{1} & \text { with probability } \theta_{1} \mathrm{~d} t \\
\min \left(a_{2} X_{2}, b_{2}\right):=Y_{2} & \text { with probability } \theta_{2} \mathrm{~d} t \\
Y_{1}+Y_{2} & \text { with probability } \theta_{12} \mathrm{~d} t\end{cases}
\end{aligned}
$$

We assume an insurer's utility function $u: R \rightarrow R, u \in C^{2}(R)$, with $u^{\prime}>0$ and $u^{\prime \prime}<0$. In particular, let ube defined as follows:

$$
u(x)=-\frac{1}{\beta} \mathrm{e}^{-\beta x}, \beta>0, x \in R
$$


The insurer looks for an optimal control strategy so as to maximize the expected utility of the terminal surplus under the initial condition regarding the $x$ state at time $t$. We consider the following value function:

$$
V(t, x)=\sup _{(a, b) \in A} E[u(R(T)) \mid R(t)=x], t \in[0, T),
$$

with the boundary condition

$$
V(T, x)=u(x)
$$

\section{The Infinitesimal Generator and the HJB Equation}

We are able to find the infinitesimal generator for the process $R(t)$ and for the function $V$. This allows us to write the HJB equation; we prove the following theorem:

Theorem 1. Let $\mathrm{V}$ be defined by (10) and let $V \in C^{1,1}([0, T] \times R)$. Therefore, $\mathrm{V}$ satisfies the following HJB equation:

$$
\begin{aligned}
& \sup _{(a, b) \in A}\left\{\partial V(t, x) / \partial t+P \partial V(t, x) / \partial x+\theta_{1} \int_{0}^{\frac{b_{1}}{a_{1}}} V\left(t, x-a_{1} s\right) \mathrm{d} F_{1}(s)\right. \\
& +\theta_{2} \int_{0}^{\frac{b_{2}}{a_{2}}} V\left(t, x-a_{2} s\right) \mathrm{d} F_{2}(s)+\theta_{12} \int_{0}^{\frac{b_{1}}{a_{1}}} \int_{0}^{\frac{b_{2}}{a_{2}}} V\left(t, x-a_{1} s-a_{2} z\right) \mathrm{d} F_{1}(s) \mathrm{d} F_{2}(z) \\
& +\left[1-F_{1}\left(\frac{b_{1}}{a_{1}}\right)\right]\left(\theta_{1} V\left(t, x-b_{1}\right)+\theta_{12} \int_{0}^{\frac{b_{2}}{a_{2}}} V\left(t, x-b_{1}-a_{2} z\right) \mathrm{d} F_{2}(z)\right) \\
& +\left[1-F_{2}\left(\frac{b_{2}}{a_{2}}\right)\right]\left(\theta_{2} V\left(t, x-b_{2}\right)+\theta_{12} \int_{0}^{\frac{b_{1}}{a_{1}}} V\left(t, x-a_{1} s-b_{2}\right) \mathrm{d} F_{1}(s)\right) \\
& \left.+\theta_{12} V\left(t, x-b_{1}-b_{2}\right)\left[1-F_{1}\left(\frac{b_{1}}{a_{1}}\right)\right]\left[1-F_{2}\left(\frac{b_{2}}{a_{2}}\right)\right]-\left(\theta_{1}+\theta_{2}+\theta_{12}\right) V(t, x)\right\}=0
\end{aligned}
$$

Proof. We derive the following infinitesimal generator $L_{t} V(t, x)$ for the process $R(t)$ and for the function $V$. The procedure is similar to that used in [7] and [8].

$$
L_{t} V(t, x)=\lim _{h \bigotimes} \frac{1}{h} E[(V(t+h, R(t+h))-V(t, R(t)) \mid R(t)=x)] .
$$

Remembering (7) and (8), it results:

$$
\begin{aligned}
L_{t} V(t, x)= & \lim _{h \searrow 0} \frac{1}{h}[V(t+h, x+P h)-V(t, x)]\left(1-\left(\theta_{1}+\theta_{2}+\theta_{12}\right) h\right) \\
& +\lim _{h \searrow 0} \frac{1}{h} E\left[V\left(t+h, x+P h-Y_{1}\right)-V(t, x)\right] \theta_{1} h \\
& +\lim _{h \searrow 0} \frac{1}{h} E\left[V\left(t+h, x+P h-Y_{2}\right)-V(t, x)\right] \theta_{2} h \\
& +\lim _{h \searrow 0} \frac{1}{h} E\left[V\left(t+h, x+P h-Y_{1}-Y_{2}\right)-V(t, x)\right] \theta_{12} h,
\end{aligned}
$$

where we have:

$$
\begin{aligned}
& \lim _{h \searrow 0} \frac{1}{h}[V(t+h, x+P h)-V(t, x)]\left(1-\left(\theta_{1}+\theta_{2}+\theta_{12}\right) h\right) \\
& =\lim _{\sqrt{1+P^{2}} h:=\bar{h} \searrow 0} \frac{V\left(t+\frac{\bar{h}}{\sqrt{1+P^{2}}}, x+\frac{\bar{h}}{\sqrt{1+P^{2}}} P\right)-V(t, x)}{\bar{h}} \sqrt{1+P^{2}}+0 \\
& =\frac{\partial V(t, x)}{\partial t}+\frac{\partial V(t, x)}{\partial x} P ;
\end{aligned}
$$




$$
\begin{aligned}
& \lim _{h \searrow 0} \frac{1}{h} E\left[V\left(t+h, x+P h-Y_{i}\right)-V(t, x)\right] \theta_{i} h \\
& =\theta_{i} \int_{0}^{\frac{b_{i}}{a_{i}}} V\left(t, x-a_{i} s\right) \mathrm{d} F_{i}(s)+\theta_{i} V\left(t, x-b_{i}\right)\left[1-F_{i}\left(\frac{b_{i}}{a_{i}}\right)\right]-\theta_{i} V(t, x), i=1,2 \text {; } \\
& \lim _{h \backslash 0} \frac{1}{h} E\left[V\left(t+h, x+P h-Y_{1}-Y_{2}\right)-V(t, x)\right] \theta_{12} h \\
& =\theta_{12}\left[\int_{0}^{\frac{b_{1}}{a_{1}}} \int_{0}^{\frac{b_{2}}{a_{2}}} V\left(t, x-a_{1} s-a_{2} z\right) \mathrm{d} F_{1}(s) \mathrm{d} F_{2}(z)\right. \\
& +\int_{0}^{\frac{b_{1}}{a_{1}}} V\left(t, x-a_{1} s-b_{2}\right)\left[1-F_{2}\left(\frac{b_{2}}{a_{2}}\right)\right] \mathrm{d} F_{1}(s) \\
& +\int_{0}^{\frac{b_{2}}{a_{2}}} V\left(t, x-b_{1}-a_{2} z\right)\left[1-F_{1}\left(\frac{b_{1}}{a_{1}}\right)\right] \mathrm{d} F_{2}(z) \\
& \left.+V\left(t, x-b_{1}-b_{2}\right)\left[1-F_{1}\left(\frac{b_{1}}{a_{1}}\right)\right]\left[1-F_{2}\left(\frac{b_{2}}{a_{2}}\right)\right]-V(t, x)\right]
\end{aligned}
$$

and therefore we find:

$$
\begin{aligned}
L_{t} V(t, x)= & \frac{\partial V(t, x)}{\partial t}+\frac{\partial V(t, x)}{\partial x} P+\theta_{1} \int_{0}^{\frac{b_{1}}{a_{1}}} V\left(t, x-a_{1} s\right) \mathrm{d} F_{1}(s) \\
& +\theta_{2} \int_{0}^{\frac{b_{2}}{a_{2}}} V\left(t, x-a_{2} s\right) \mathrm{d} F_{2}(s)+\theta_{12} \int_{0}^{\frac{b_{1}}{a_{1}}} \int_{0}^{\frac{b_{2}}{a_{2}}} V\left(t, x-a_{1} s-a_{2} z\right) \mathrm{d} F_{1}(s) \mathrm{d} F_{2}(z) \\
& +\left[1-F_{1}\left(\frac{b_{1}}{a_{1}}\right)\right]\left(\theta_{1} V\left(t, x-b_{1}\right)+\theta_{12} \int_{0}^{\frac{b_{2}}{a_{2}}} V\left(t, x-b_{1}-a_{2} z\right) \mathrm{d} F_{2}(z)\right) \\
& +\left[1-F_{2}\left(\frac{b_{2}}{a_{2}}\right)\right]\left(\theta_{2} V\left(t, x-b_{2}\right)+\theta_{12} \int_{0}^{\frac{b_{1}}{a_{1}}} V\left(t, x-a_{1 s}-b_{2}\right) \mathrm{d} F_{1}(s)\right) \\
& \left.+\theta_{12} V\left(t, x-b_{1}-b_{2}\right)\left[1-F_{1}\left(\frac{b_{1}}{a_{1}}\right)\right]\left[1-F_{2}\left(\frac{b_{2}}{a_{2}}\right)\right]-\left(\theta_{1}+\theta_{2}+\theta_{12}\right) V(t, x)\right] ;
\end{aligned}
$$

the Equation (12) is therefore fulfilled by $V$.

As we specified before, we assume the utility function (9), inspired by [1] [4] [8]-[10], we look for a solution of the problem (10), with the condition (11) of the form:

$$
V(t, x)=-\frac{1}{\beta} \mathrm{e}^{-\beta(x-Q(t))},
$$

with $Q(T)=0$.

We note that:

$$
\begin{gathered}
\frac{\partial V(t, x)}{\partial t}=\beta Q^{\prime}(t) V(t, x) \\
\frac{\partial V(t, x)}{\partial x}=-\beta V(t, x) \\
V(t, x-k)=V(t, x) \mathrm{e}^{\beta k}
\end{gathered}
$$

Therefore, (12) can be written as follows: 


$$
\begin{aligned}
& \sup _{(a, b) \in A}\left\{-V(t, x)\left[-\beta Q^{\prime}(t)+\beta P-\theta_{1} \int_{0}^{\frac{b_{1}}{a_{1}}} \mathrm{e}^{\beta a_{1} s} \mathrm{~d} F_{1}(s)\right.\right. \\
& -\theta_{2} \int_{0}^{\frac{b_{2}}{a_{2}}} \mathrm{e}^{\beta a_{2} z} \mathrm{~d} F_{2}(z)-\theta_{12} \int_{0}^{\frac{b_{1}}{a_{1}}} \int_{0}^{\frac{b_{2}}{a_{2}}} \mathrm{e}^{\beta a_{1} s+\beta a_{2} z} \mathrm{~d} F_{1}(s) \mathrm{d} F_{2}(z) \\
& -\left[1-F_{1}\left(\frac{b_{1}}{a_{1}}\right)\right]\left(\theta_{1} \mathrm{e}^{\beta b_{1}}+\theta_{12} \int_{0}^{\frac{b_{2}}{a_{2}}} \mathrm{e}^{\beta b_{1}+\beta a_{2} z} \mathrm{~d} F_{2}(z)\right) \\
& -\left[1-F_{2}\left(\frac{b_{2}}{a_{2}}\right)\right]\left(\theta_{2} \mathrm{e}^{\beta b_{2}}+\theta_{12} \int_{0}^{\frac{b_{1}}{a_{1}}} \mathrm{e}^{\beta a_{1} s+\beta b_{2}} \mathrm{~d} F_{1}(s)\right) \\
& \left.\left.-\theta_{12} \mathrm{e}^{\beta b_{1}+\beta b_{2}}\left[1-F_{1}\left(\frac{b_{1}}{a_{1}}\right)\right]\left[1-F_{2}\left(\frac{b_{2}}{a_{2}}\right)\right]+\left(\theta_{1}+\theta_{2}+\theta_{12}\right)\right]\right\}=0 .
\end{aligned}
$$

Observing that:

$$
\int_{0}^{\frac{b_{i}}{a_{i}}} \mathrm{e}^{\beta a_{i} s} \mathrm{~d} F_{i}(s)=1-\mathrm{e}^{\beta b_{i}}\left[1-F_{i}\left(\frac{b_{i}}{a_{i}}\right)\right]+\beta a_{i} \int_{0}^{\frac{b_{i}}{a_{i}}} \mathrm{e}^{\beta a_{i} s}\left(1-F_{i}(s)\right) \mathrm{d} s, \quad i=1,2,
$$

it follows that Equation (13), dividing by $-V(t, x)>0$ and remembering (6), can be written as follows:

$$
\begin{aligned}
& \sup _{(a, b) \in A}\left\{-\beta Q^{\prime}(t)+\beta \sum_{i=1}^{2}\left[\left(\theta_{i}+\theta_{12}\right) \mu_{i}\left(\eta_{i}-\gamma_{i}\right)+\left(1+\gamma_{i}\right)\left(\theta_{i}+\theta_{12}\right) a_{i} \int_{0}^{\frac{b_{i}}{a_{i}}}\left(1-F_{i}(s)\right) \mathrm{d} s\right]\right. \\
& -\left(\theta_{1}+\theta_{12}\right) \beta a_{1} \int_{0}^{\frac{b_{1}}{a_{1}}} \mathrm{e}^{\beta a_{1} s}\left(1-F_{1}(s)\right) \mathrm{d} s-\left(\theta_{2}+\theta_{12}\right) \beta a_{2} \int_{0}^{\frac{b_{2}}{a_{2}}} \mathrm{e}^{\beta a_{2} z}\left(1-F_{2}(z)\right) \mathrm{d} z \\
& \left.-\theta_{12} \beta^{2} a_{1} a_{2} \int_{0}^{\frac{b_{1}}{a_{1}}} \mathrm{e}^{\beta a_{1} s}\left(1-F_{1}(s)\right) \mathrm{d} s \int_{0}^{\frac{b_{2}}{a_{2}}} \mathrm{e}^{\beta a_{2} z}\left(1-F_{2}(z)\right) \mathrm{d} z\right\}=0 .
\end{aligned}
$$

In the particular case where $b_{i}=+\infty, i=1,2$, we obtain the case study regarding the only proportional reinsurance, as analyzed in [4] [8]; under this assumption, Equation (6), remembering (3) and the assumptions made about the random variables $X_{i}$, gives:

$$
P=\sum_{i=1}^{2}\left(c_{i}-\left(\theta_{i}+\theta_{12}\right)\left(1+\gamma_{i}\right)\left(1-a_{i}\right) \mu_{i}\right) .
$$

Therefore, (13) becomes:

$$
\begin{aligned}
& \sup _{(a,+\infty) \in A}\left\{-V(t, x)\left[-\beta Q^{\prime}(t)+\beta \sum_{i=1}^{2}\left(c_{i}-\left(\theta_{i}+\theta_{12}\right)\left(1+\gamma_{i}\right)\left(1-a_{i}\right) \mu_{i}\right)\right.\right. \\
& \left.\left.-\theta_{1} \int_{0}^{+\infty} \mathrm{e}^{\beta a_{1} s} \mathrm{~d} F_{1}(s)-\theta_{2} \int_{0}^{+\infty} \mathrm{e}^{\beta a_{2} z} \mathrm{~d} F_{2}(z)-\theta_{12} \int_{0}^{+\infty} \int_{0}^{+\infty} \mathrm{e}^{\beta a_{1} s+\beta a_{2} z} \mathrm{~d} F_{1}(z) \mathrm{d} F_{2}(z)+\left(\theta_{1}+\theta_{2}+\theta_{12}\right)\right]\right\}=0 .
\end{aligned}
$$

It is obviously that Equation (15) is the same equation found in [8]; furthermore Equation (15) divided by $V(t, x)<0$ coincides with Equation (3.4) of [1] with $r=0$ and $\delta\left(q_{1}, q_{2}\right)$ calculated by means of the expected value principle.

In the particular case where $a_{i}=1, i=1,2$, we have the only excess of loss reinsurance case. Under this assumption, Equation (13), written on the form (14), is:

$$
\begin{aligned}
& \sup _{(1, b) \in A}\left\{-\beta Q^{\prime}(t)+\beta \sum_{i=1}^{2}\left[\left(\theta_{i}+\theta_{12}\right) \mu_{i}\left(\eta_{i}-\gamma_{i}\right)+\left(1+\gamma_{i}\right)\left(\theta_{i}+\theta_{12}\right) \int_{0}^{b_{i}}\left(1-F_{i}(s)\right) \mathrm{d} s\right]\right. \\
& -\left(\theta_{1}+\theta_{12}\right) \beta \int_{0}^{b_{1}} \mathrm{e}^{\beta s}\left(1-F_{1}(s)\right) \mathrm{d} s-\left(\theta_{2}+\theta_{12}\right) \beta \int_{0}^{b_{2}} \mathrm{e}^{\beta z}\left(1-F_{2}(z)\right) \mathrm{d} z \\
& \left.-\theta_{12} \beta^{2} \int_{0}^{b_{1}} \mathrm{e}^{\beta s}\left(1-F_{1}(s)\right) \mathrm{d} s \int_{0}^{b_{2}} \mathrm{e}^{\beta z}\left(1-F_{2}(z)\right) \mathrm{d} z\right\}=0 .
\end{aligned}
$$


In the following section we consider this case.

\section{The Excess of Loss Reinsurance Case}

We face the problem (16), with condition (11), that is $Q(T)=0$. We write (16) as follows

$$
\sup _{(1, b) \in A} g\left(b_{1}, b_{2}\right)=0
$$

with conditions:

$$
b_{i} \geq 0, i=1,2
$$

and

$$
Q(T)=0
$$

We have:

$$
\frac{\partial g\left(b_{1}, b_{2}\right)}{\partial b_{1}}=\beta\left(1-F_{1}\left(b_{1}\right)\right)\left[\left(\theta_{1}+\theta_{12}\right)\left(1+\gamma_{1}\right)-\mathrm{e}^{\beta b_{1}}\left(\left(\theta_{1}+\theta_{12}\right)+\beta \theta_{12} \int_{0}^{b_{2}} \mathrm{e}^{\beta z}\left(1-F_{2}(z)\right) \mathrm{d} z\right)\right]
$$

and

$$
\frac{\partial g\left(b_{1}, b_{2}\right)}{\partial b_{2}}=\beta\left(1-F_{2}\left(b_{2}\right)\right)\left[\left(\theta_{2}+\theta_{12}\right)\left(1+\gamma_{2}\right)-\mathrm{e}^{\beta b_{2}}\left(\left(\theta_{2}+\theta_{12}\right)+\beta \theta_{12} \int_{0}^{b_{1}} \mathrm{e}^{\beta s}\left(1-F_{1}(s)\right) \mathrm{d} s\right)\right]
$$

from which we deduce that, at the points where the gradient of $g$ is zero, the Hessian matrix of $g\left(b_{1}, b_{2}\right)$ is negative defined. The relative proof can be immediately obtained using results in [3]. We can therefore search the solution of the problem (17), with conditions (18), looking for the solutions of the following system:

$$
\left\{\begin{array}{l}
\frac{\partial g\left(b_{1}, b_{2}\right)}{\partial b_{i}}=-\lambda_{i} \leq 0, i=1,2 \\
b_{i} \lambda_{i}=0, i=1,2 \\
b_{i} \geq 0, i=1,2
\end{array}\right.
$$

that is, letting $\xi_{i}=\frac{\lambda_{i}}{\beta\left(1-F_{i}\left(b_{i}\right)\right)}$, and remembering (20) and (21), we look for the solution of the system:

$$
\left\{\begin{array}{l}
\left(\theta_{1}+\theta_{12}\right)\left(1+\gamma_{1}\right)-\mathrm{e}^{\beta b_{1}}\left(\left(\theta_{1}+\theta_{12}\right)+\beta \theta_{12} \int_{0}^{b_{2}} \mathrm{e}^{\beta z}\left(1-F_{2}(z)\right) \mathrm{d} z\right)=-\xi_{1} \leq 0 \\
\left(\theta_{2}+\theta_{12}\right)\left(1+\gamma_{2}\right)-\mathrm{e}^{\beta b_{2}}\left(\left(\theta_{2}+\theta_{12}\right)+\beta \theta_{12} \int_{0}^{b_{1}} \mathrm{e}^{\beta s}\left(1-F_{1}(s)\right) \mathrm{d} s\right)=-\xi_{2} \leq 0 \\
b_{i} \xi_{i}=0, i=1,2 \\
b_{i} \geq 0, i=1,2 .
\end{array}\right.
$$

The solutions can be of the following four kinds:

$$
\begin{aligned}
& \text { (I) } b_{1}^{*}=b_{2}^{*}=0 \\
& \text { (II) } b_{1}^{*}=0 \text { and } b_{2}^{*}>0 \\
& \text { (III) } b_{1}^{*}>0 \text { and } b_{2}^{*}=0 \\
& \text { (IV) } b_{1}^{*}>0 \text { and } b_{2}^{*}>0 .
\end{aligned}
$$

We observe that the solution $(I) b_{1}^{*}=b_{2}^{*}=0$ does not exist. Indeed, the system (22) gives:

$$
\left\{\begin{array}{l}
\left(\theta_{1}+\theta_{12}\right) \gamma_{1} \leq 0 \\
\left(\theta_{2}+\theta_{12}\right) \gamma_{2} \leq 0
\end{array}\right.
$$

that is impossible. 
According to results in [3], we have:

(II) $b_{1}^{*}=0$ and $b_{2}^{*}>0$. The system (22) can be written as:

$$
\left\{\begin{array}{l}
\left(\theta_{1}+\theta_{12}\right) \gamma_{1}-\theta_{12} \beta \int_{0}^{b_{2}} \mathrm{e}^{\beta z}\left(1-F_{2}(z)\right) \mathrm{d} z \leq 0 \\
\left(\theta_{2}+\theta_{12}\right)\left[\left(1+\gamma_{2}\right)-\mathrm{e}^{\beta b_{2}}\right]=0 \\
\xi_{2}=0 \\
b_{2}>0
\end{array}\right.
$$

from which, if:

$$
\left(\theta_{1}+\theta_{12}\right) \gamma_{1} \leq \beta \theta_{12} \int_{0}^{\frac{1}{\beta} \ln \left(1+\gamma_{2}\right)} \mathrm{e}^{\beta z}\left(1-F_{2}(z)\right) \mathrm{d} z,
$$

we have:

$$
b_{1}^{*}=0, b_{2}^{*}=\frac{\ln \left(1+\gamma_{2}\right)}{\beta}
$$

(III) $b_{1}^{*}>0$ and $b_{2}^{*}=0$. In a similar way to (II), if:

$$
\left(\theta_{2}+\theta_{12}\right) \gamma_{2} \leq \beta \theta_{12} \int_{0}^{\frac{1}{\beta} \ln \left(1+\gamma_{1}\right)} \mathrm{e}^{\beta s}\left(1-F_{1}(s)\right) \mathrm{d} s,
$$

we have:

$$
b_{1}^{*}=\frac{\ln \left(1+\gamma_{1}\right)}{\beta}, b_{2}^{*}=0
$$

(IV) $b_{1}^{*}>0$ and $b_{2}^{*}>0$. The system (22) gives:

$$
\left\{\begin{array}{l}
\left(\theta_{1}+\theta_{12}\right)\left(1+\gamma_{1}\right)=\mathrm{e}^{\beta b_{1}^{*}}\left(\left(\theta_{1}+\theta_{12}\right)+\theta_{12} \beta \int_{0}^{b_{2}^{*}} \mathrm{e}^{\beta z}\left(1-F_{2}(z)\right) \mathrm{d} z\right) \\
\left(\theta_{2}+\theta_{12}\right)\left(1+\gamma_{2}\right)=\mathrm{e}^{\beta b_{2}^{*}}\left(\left(\theta_{2}+\theta_{12}\right)+\theta_{12} \beta \int_{0}^{b_{1}^{*}} \mathrm{e}^{\beta s}\left(1-F_{1}(s)\right) \mathrm{d} s\right) \\
\xi_{1}=\xi_{2}=0 \\
b_{i} \geq 0, i=1,2 .
\end{array}\right.
$$

In [3], it is proved that under the assumption that both (23) and (24) are not satisfied, that is

$$
\left(\theta_{1}+\theta_{12}\right) \gamma_{1}>\beta \theta_{12} \int_{0}^{\frac{1}{\beta} \ln \left(1+\gamma_{2}\right)} \mathrm{e}^{\beta z}\left(1-F_{2}(z)\right) \mathrm{d} z
$$

and

$$
\left(\theta_{2}+\theta_{12}\right) \gamma_{2}>\beta \theta_{12} \int_{0}^{0 \frac{1}{\beta} \ln \left(1+\gamma_{1}\right)} \mathrm{e}^{\beta s}\left(1-F_{1}(s)\right) \mathrm{d} s,
$$

the optimal strategy $\left(b_{1}^{*}, b_{2}^{*}\right)$, fulfilling (25), exists with

$$
0<b_{1}^{*}<\frac{1}{\beta} \ln \left(1+\gamma_{1}\right) .
$$

We observe that, from (28), being true also (27) and since $b_{2}^{*}$ fulfilled the system (25), it results that:

$$
0<b_{2}^{*}<\frac{1}{\beta} \ln \left(1+\gamma_{2}\right) .
$$

Finally, we recall that (23) and (24) are incompatible (see [3]).

We are so able to find the value function, substituting the optimal strategy in (17), that is in (16), and obtaining $Q(t)$ with the condition $Q(T)=0$. If (23) is fulfilled, we have: 


$$
Q(t):=Q_{1}(t)=\left[\sum_{i=1}^{2}\left(\theta_{i}+\theta_{12}\right) \mu_{i}\left(\gamma_{i}-\eta_{i}\right)-\left(\theta_{2}+\theta_{12}\right) \int_{0}^{\frac{1}{\beta} \ln \left(1+\gamma_{2}\right)}\left(1+\gamma_{2}-\mathrm{e}^{\beta z}\right)\left(1-F_{2}(z)\right) \mathrm{d} z\right](T-t) ;
$$

if (24) is fulfilled, it results:

$$
Q(t):=Q_{2}(t)=\left[\sum_{i=1}^{2}\left(\theta_{i}+\theta_{12}\right) \mu_{i}\left(\gamma_{i}-\eta_{i}\right)-\left(\theta_{1}+\theta_{12}\right) \int_{0}^{\frac{1}{\beta} \ln \left(1+\gamma_{1}\right)}\left(1+\gamma_{1}-\mathrm{e}^{\beta s}\right)\left(1-F_{1}(s)\right) \mathrm{d} s\right](T-t) ;
$$

if (26) and (27) are at the same time fulfilled, we have:

$$
\begin{aligned}
Q(t): & Q_{3}(t)=\left[\sum_{i=1}^{2}\left(\theta_{i}+\theta_{12}\right) \mu_{i}\left(\gamma_{i}-\eta_{i}\right)\right. \\
& -\left(\theta_{1}+\theta_{12}\right) \int_{0}^{b_{1}^{*}}\left(1+\gamma_{1}-\mathrm{e}^{\beta s}\right)\left(1-F_{1}(s)\right) \mathrm{d} s \\
& -\left(\theta_{2}+\theta_{12}\right) \int_{0}^{b_{2}^{*}}\left(1+\gamma_{2}-\mathrm{e}^{\beta z}\right)\left(1-F_{2}(z)\right) \mathrm{d} z \\
& \left.+\theta_{12} \beta \int_{0}^{b_{1}^{*}} \mathrm{e}^{\beta s}\left(1-F_{1}(s)\right) \mathrm{d} s \int_{0}^{b_{2}^{*}} \mathrm{e}^{\beta z}\left(1-F_{2}(z)\right) \mathrm{d} z\right](T-t) .
\end{aligned}
$$

The results obtained in this section are collected within the following theorem.

Theorem 2. The optimal strategy $\left(b_{1}^{*}, b_{2}^{*}\right)$ concerning a whole excess of loss reinsurance and the corresponding value function are the following:

- if

$$
\left(\theta_{1}+\theta_{12}\right) \gamma_{1} \leq \beta \theta_{12} \int_{0}^{\frac{1}{\beta} \ln \left(1+\gamma_{2}\right)} \mathrm{e}^{\beta z}\left(1-F_{2}(z)\right) \mathrm{d} z
$$

it is

$$
\left(q_{1}^{*}=0, \quad q_{2}^{*}=\frac{1}{\beta} \ln \left(1+\gamma_{2}\right)\right)
$$

and

$$
V(t, x)=-\frac{1}{\beta} \mathrm{e}^{-\beta\left(x-Q_{1}(t)\right)}
$$

where $Q_{1}(t)$ is given by (30);

- if

$$
\left(\theta_{2}+\theta_{12}\right) \gamma_{2} \leq \beta \theta_{12} \int_{0}^{\frac{1}{\beta} \ln \left(1+\gamma_{1}\right)} \mathrm{e}^{\beta s}\left(1-F_{1}(s)\right) \mathrm{d} s
$$

it is

$$
\left(q_{1}^{*}=\frac{1}{\beta} \ln \left(1+\gamma_{1}\right), q_{2}^{*}=0\right)
$$

and

$$
V(t, x)=-\frac{1}{\beta} \mathrm{e}^{-\beta\left(x-Q_{2}(t)\right)}
$$

where $Q_{2}(t)$ is given by (31);

- if

$$
\left(\theta_{1}+\theta_{12}\right) \gamma_{1}>\beta \theta_{12} \int_{0}^{\frac{1}{\beta} \ln \left(1+\gamma_{2}\right)} \mathrm{e}^{\beta z}\left(1-F_{2}(z)\right) \mathrm{d} z
$$

and 


$$
\left(\theta_{2}+\theta_{12}\right) \gamma_{2}>\beta \theta_{12} \int_{0}^{\frac{1}{\beta} \ln \left(1+\gamma_{1}\right)} \mathrm{e}^{\beta s}\left(1-F_{1}(s)\right) \mathrm{d} s
$$

it is

$$
\left(0<q_{1}^{*}<\frac{1}{\beta} \ln \left(1+\gamma_{1}\right), \quad 0<q_{2}^{*}<\frac{1}{\beta} \ln \left(1+\gamma_{2}\right)\right)
$$

and

$$
V(t, x)=-\frac{1}{\beta} \mathrm{e}^{-\beta\left(x-Q_{3}(t)\right)}
$$

where $Q_{3}(t)$ is given by (32).

\section{Acknowledgements}

We thank the Editor and the Referees for their comments.

\section{References}

[1] Liang, Z. and Guo, Y. (2011) Optimal Combining Quota-Share and Excess of Loss Reinsurance to Maximize the Expected Utility. Journal of Applied Mathematics and Computing (JAMC), 36, 11-25. http://dx.doi.org/10.1007/s12190-010-0385-8

[2] Schmidli, H. (2001) Optimal Proportional Reinsurance Policies in a Dynamyc Setting. Scandinavian Actuarial Journal, 1, 55-68. http://dx.doi.org/10.1080/034612301750077338

[3] Centeno, M.L. (2005) Dependent Risks and Excess of Loss Reinsurance. Insurance: Mathematics and Economics, 37, 229-238. http://dx.doi.org/10.1016/j.insmatheco.2004.12.001

[4] Liang, Z. and Yuen, K.C. (2016) Optimal Dynamic Reinsurance with Dependent Risks: Variance Premium Principle. Scandinavian Actuarial Journal, 1, 18-36. http://dx.doi.org/10.1080/03461238.2014.892899

[5] Yuen, K.C., Liang, Z. and Zhou, M. (2015) Optimal Proportional Reinsurance with Common Shock Dependence. Insurance: Mathematics and Economics, 4, 1-13. http://dx.doi.org/10.1016/j.insmatheco.2015.04.009

[6] Hu, F. and Yuen, K.C. (2012) Optimal Proportional Reinsurance under Dependent Risks. Journal of Systems Science and Complexity, 25, 1171. http://dx.doi.org/10.1007/s11424-012-1045-x

[7] Cetin, U. (2014) An Introduction to Markov Processes and Their Applications in Mathematical Economics. Lecture Notes. Department of Statistic, London School of Economics and Political Science, London.

[8] Gosio, C., Lari, E.C. and Ravera, M. (2015) Optimal Proportional Reinsurance in a Bivariate Risk Model. Modern Economy, 6, 664-671. http://dx.doi.org/10.4236/me.2015.66062

[9] Lin, X. and Li, Y. (2011) Optimal Reinsurance and Investment for a Jump Diffusion Risk Process under the CEV Model. North American Actuarial Journal, 15, 3, 417-431. http://dx.doi.org/10.1080/10920277.2011.10597628

[10] Johnson, N.L., Kotz, S. and Balakrishnan, N. (1997) Discrete Multivariate Distributions. Wiley \& Sons, New York. 